\title{
PRODUCTOS DE APOYO PARA PACIENTE CON AMPUTACIÓN TRANSMETACARPIANA
}

\author{
SUPPORT PRODUCTS FOR PATIENT WITHTRANSMETACARPAL AMPUTATION
}

\section{Mariana Flores E. ${ }^{1}$, Agustina Galarza C. ${ }^{2}$}

\begin{abstract}
Resumen:
Propósito: Analizar propiedades funcionales y antropométricas de los PA, criterios de selección de diseño, procesos de confección y materiales de fabricación, para analizar las ventajas y/o desventajas que proporcionan y el impacto de esto sobre la CV, ya que los PA disponibles en el mercado no siempre se adaptan a requerimientos del individuo. Se demostrará a través de la presentación de un caso clínico con amputación transmetacarpiana izquierda, de 60 años sufrida el 24/11/2012. Metodología y Materiales: Mediciones antropométricas elementos termoplásticos y de descarte. Resultados: A partir del análisis de propiedades y características de los PA se podría establecer la forma en la cual éstos inciden en la CV de las personas en cuanto a confort, seguridad e independencia funcional, y a partir de ello conocer el grado de satisfacción del usuario respecto a su participación ocupacional en las AVD. Conclusiones: Respecto al desarrollo de PA, se están abriendo nuevas posibilidades para la mejora de la CV de la población en general. Si bien el avance tecnológico está orientado a potenciar la autonomía de los usuarios mediante nuevas y variadas estrategias se ha ido intensificando en campos como la rehabilitación y la antropometría, es necesario en más de una ocasión incorporar acciones que permitan al paciente mejorar su economía, funcionalidad, durabilidad y adecuación estética.
\end{abstract}

Palabras clave:

Productos de Apoyo (PA); Calidad de Vida (CV); Actividades de la Vida Diaria (AVD); Antropometría; Amputación parcial de la Mano.

\begin{abstract}
Abstrac:
Purpose: Analyze SP functional and anthropometric properties, design selection criteria, preparation processes and manufacturing materials, to analyze the advantages and/or disadvantages that they have and their impact on the $L Q$, since the $S P$ available in the market do not always adapt to the individual's requirements. It will be demonstrated through the presentation of a clinical case with left transmetacarpal amputation, 60 years old, suffered on 11/24/2012. Methodology and Materials: anthropometric measures, thermoplastic and disposable items. Results: From the analysis of properties and characteristics of the SP it could be established the way in which they affect people's $L Q$ in terms of comfort, safety and functional independence, and considering this we can determine the degree of the user's satisfaction regarding occupational participation in DLA. Conclusions: Regarding the SP development, new possibilities are appearing for the improvement of general population LQ. Even though the technological advance oriented to highlight the user's autonomy through new and various strategies has been intensifying in some other fields like rehabilitation and anthropometry, it is necessary in more than one occasion to incorporate actions that allow the patient to enhance his functionality with economy criteria, functionality, durability and aesthetics as quality standards in design and construction.
\end{abstract}

Keywords:

Support Products (SP), Life Quality (LQ), Daily Life Activities (DLA), Anthropometry, Partial Amputation of the Hand.

1 Terapista Ocupacional / Universitario / Jefa del Servicio de Terapia Ocupacional de ILAR. Profesora JTP DS Carrera de Lic en Terapia Ocupacional. UNVM / Balcarce 128 BIS. Rosario. Santa Fe. Argentina. CP 2000 / Tel-Cel: 54-341 155699494 / E-mail: marianagflores@ hotmail.com

2 Estudiante de la Carrera Lic. de Terapia Ocupacional UNVM / Cursante del 5año / Alumna Becaria del Proyecto de Investigación "La Incidencia de los PA para las AVBD en la CV de las personas" / Hipólito Irigoyen 592. Córdoba. Argentina. CP 5000 / Tel-Cel: 54-351 152424196 / E-mail: agusgalarza89@gmail.com 


\section{INTRODUCCIÓN}

Al momento de satisfacer sus necesidades las personas implementan nuevos y variados procedimientos para realizar diferentes tareas, adaptándose así a las diversas y cambiantes condiciones del entorno. De esta manera se logran aprender nuevas formas de desempeño en las actividades cotidianas. Sin embargo, en ocasiones esta capacidad de adaptación para funcionar de forma autónoma se ve sobrepasada, y se torna necesario realizar un análisis exhaustivo de las condiciones que requieren ser modificadas (ya sea el medio, algunos objetos, o la modalidad de realizar convencionalmente una actividad) para propiciar un mejor desempeño. La antropometría ha hecho sus respectivos aportes a través del estudio y análisis de las medidas y dimensiones del hombre; de esta forma se convierte en una ciencia fundamental al momento de diseñar y confeccionar aquellos productos que dependerán de las dimensiones de quienes serán sus usuarios. Respecto a estas dimensiones hay dos que deben ser valoradas, tanto las estructurales como las funcionales; las primeras en relación a los segmentos corporales en sí, y las segundas tienen que ver con las medidas tomadas durante la ejecución de un movimiento determinado durante una actividad específica. Serán estas medidas las que signen el diseño del producto a partir de los segmentos estructurales requeridos para su utilización y funcionalización.

Por otro lado, los Productos de Apoyo se definen como: "cualquier producto (incluyendo dispositivos, equipo, instrumentos, tecnologías y software) fabricado especialmente o disponible en el mercado, para prevenir, compensar, controlar, mitigar o neutralizar deficiencias, limitaciones en la actividad y restricciones en la participación." (AENOR, 2007).

En la praxis de la terapia ocupacional la adaptación de las tareas y/o el entorno de desempeño, implica el diseño y elaboración de diferentes productos de variada complejidad. La American Occupational Therapy Association plantea que la Terapia Ocupacional es:

Terapia Ocupacional es el uso terapéutico de las actividades de autocuidado, trabajo y juego para incrementar la función independiente, mejorar el desarrollo y prevenir la discapacidad. Puede incluir la adaptación de las tareas o el ambiente para lograr la máxima independencia y mejorar la calidad de vida. (Polonio L., B.; Durante M., P.; Noya A., B., 2001).

En el presente trabajo se expondrán PA diseñados pertinentes al área de las $A B V D$, más precisamente de alimentación; y se pretende determinar y analizar las propiedades funcionales y adecuación antropométrica de los mismos, criterios de selección de diseño, confección y materiales de fabricación. En base a esto, determinar las ventajas y/o desventajas que proporcionan y el impacto de esto sobre la calidad de vida del usuario destinatario.

Se entiende por CV "a la percepción del individuo y el nivel de satisfacción que tiene en relación a su situación física, estado emocional, su vida de relación, y el sentido que le atribuye a la misma de acuerdo a sus objetivos, expectativas, estándares y preocupaciones". (OMS, 1994).

Si bien ISO ofrece una codificación y clasificación normatizada de los PA según su propósito, no se incluyen ni se describen las propiedades de los mismos como tampoco la incidencia de éstos en la calidad de vida, a pesar de sus aportes en el desempeño funcional.

\section{Objetivos}

- Analizar las características de los diferentes PA confeccionados y determinar su incidencia en la calidad de vida del usuario.

- Valorar selectivamente los PA confeccionados a fin de precisar las propiedades de los materiales elegidos, su costo, el proceso de diseño, el uso y los sucesivos retoques planteados.

- Identificar el grado de aceptación y satisfacción, y la regularidad del uso del usuario respecto a los PA confeccionados.

- Reportar las desventajas y fallas de diseño y confección de los PA propuestos. 


\section{Metodología}

Respecto a los PA propuestos y elaborados se analizarán: los objetivos que deben cumplir; los criterios de selección de diseño y confección; las características de los materiales (como maleabilidad, resistencia, tiempo de vida útil, etc.); los costos y la accesibilidad; la complejidad en la confección, en el uso y en el cuidado; las habilidades de desempeño que se requieren para su uso, o las que compensa o sustituye; y la adaptación del diseño de los PA confeccionados a las características antropométricas del usuario.

En relación al usuario se analizarán: las ventajas y desventajas funcionales; la comprensión de la utilidad, uso y características de los PA; la adaptación/aceptación de la utilización de PA; la regularidad de uso; las ventajas/desventajas, seguridad, independencia y confort percibido por el usuario.

A su vez se tomarán medidas antropométricas del muñón del usuario, debido a que estas dimensiones son de gran importancia al momento de diseñar y confeccionar los PA que manipulará, y de esta forma poder establecer si se adecuan o no a los estándares de confort esperados por el usuario.

En el presente trabajo se utilizará la norma "ISO 9999:2007 -Productos de Apoyo para personas con discapacidad. Clasificación y Terminología-" (UNE-EN ISO 9999. (2007). Productos de apoyo para personas con discapacidad. Clasificación y terminología. (ISO 9999:2007). 4ta edición. BOE. España) para detallar los PA que se mencionarán; la misma ofrece una tipificación que se basa en el propósito o función de los productos y los organiza en pares de códigos a los fines de universalizarlos. Según esta normativa, los PA confeccionados corresponden al nivel de clase 15 "Productos de apoyo para las actividades domésticas", sub clase 09 "Productos de apoyo para comer y beber", división "adaptadores de agarre y accesorios". Sin embargo, siendo esta codificación insuficiente para especificar los PA diseñados y confeccionados, se deriva a códigos que más se adecuan, 2418 06. Considerando, nivel de clase 24 "Productos de apoyo para la manipulación de objetos y dispositivos", sub clase 18 "Productos de apoyo para compensar y/o reemplazar las funciones del brazo, mano y/o dedos", división 06 "Adaptadores de agarre y accesorios", que se refiere a los dispositivos que se añaden a un objeto para ayudar a agarrarlo.

\section{Desarrollo}

Teniendo en cuenta que para los casos clínicos como el que se presenta, la alternativa más sugerida es "el cuchillo-tenedor" puesto que se utilizaría con el miembro superior no afectado facilitando la ejecución de la actividad de alimentación. Esta opción nunca fue considerada dado que el objetivo primordial es la de lograr el máximo de la funcionalidad de ambos miembros superiores.

Es en este sentido que se deben tener en cuenta las medidas antropométricas del usuario al momento de diseñar y confeccionar un PA, a modo de garantizar no solo que pueda utilizar el PA correctamente según su fin, sino también respetando los ángulos de confort funcional evitando dañar estructuras adyacentes.

Exponemos a continuación el caso clínico de GO, de 60 años de edad, sexo femenino, dominancia diestra cuya historia ocupacional reciente se desarrollaba en una carnicería con múltiples tareas. El 14 de octubre de 2012 sufre un evento en su espacio laboral de causa traumática ocasionando amputación transmetacarpiana izquierda por lo que recibe la práctica quirúrgica oportuna inmediata y es derivada a tratamiento terapéutico.

En diciembre de 2012, ingresa al servicio de Terapia Ocupacional donde recibe abordaje específico del muñón optimizando al máximo la función de su pulgar izquierdo como así también la del muñón correspondiente al $5^{\circ}$ rayo y se suman acciones para mejorar su autonomía personal; una de ellas fue la alimentación y uso de cubiertos para dicha actividad diaria básica. Acorde a su demanda e inquietud se fueron planteando diferentes opciones de diseño y materiales.

Por lo detallado se dispone una diversidad de intervenciones a los fines de adecuar las condiciones funcionales y el requerimiento de la actividad mencionada. (Ver Tabla 1). 
Tabla $N^{\circ} 1$

-Análisis comparativo de los diferentes PA diseñados y confeccionados para el caso clínico presentado

\begin{tabular}{|c|c|c|c|c|c|}
\hline \multicolumn{2}{|c|}{ PA Criterios } & $\begin{array}{c}\text { Primer PA } \\
\text { confeccionado }\end{array}$ & $\begin{array}{c}\text { Segundo PA } \\
\text { confeccionado }\end{array}$ & $\begin{array}{c}\text { Tercer PA } \\
\text { confeccionado }\end{array}$ & $\begin{array}{c}\text { Cuarto PA } \\
\text { confeccionado }\end{array}$ \\
\hline \multicolumn{2}{|c|}{ Material } & $\begin{array}{l}\text { Termoplástico ORFIT } \\
\text { liso (no perforado), } \\
3,2 \mathrm{~mm} \text { de espesor, } \\
\text { color azul; y un tene- } \\
\text { dor convencional }\end{array}$ & $\begin{array}{l}\text { All-Fit de ORFIT, } \\
\text { abrojos y un tenedor } \\
\text { convencional }\end{array}$ & $\begin{array}{l}\text { Espuma de polieti- } \\
\text { leno (en el mercado } \\
\text { conocido como "flo- } \\
\text { ta flota") y un tene- } \\
\text { dor convencional } \\
\text { al que se le cortó la } \\
\text { totalidad del mango }\end{array}$ & $\begin{array}{l}\text { Material de descarte de } \\
\text { un tubo plástico de hilo } \\
\text { industrial, y un tenedor } \\
\text { convencional al que se } \\
\text { le cortó la totalidad } \\
\text { del mango }\end{array}$ \\
\hline \multicolumn{2}{|c|}{ Diseño } & $\begin{array}{l}\text { Se engrosó el mango } \\
\text { del tenedor con el } \\
\text { termoplástico, y se } \\
\text { anexó con el mismo } \\
\text { material un anillo }\end{array}$ & $\begin{array}{l}\text { Se engrosó el tene- } \\
\text { dor con el All-Fit, y } \\
\text { se fundió al mismo } \\
\text { los abrojos }\end{array}$ & $\begin{array}{l}\text { Al "flota flota" se le } \\
\text { realizaron dos esco- } \\
\text { taduras en dos pun- } \\
\text { tos opuestos de sus } \\
\text { cantos, y en el centro } \\
\text { se insertó el tenedor }\end{array}$ & $\begin{array}{l}\text { Al tubo se le rellenaron } \\
\text { las bordes a los fines } \\
\text { de brindar apoyo para } \\
\text { funcionalizar la "tena- } \\
\text { za", y en el centro se } \\
\text { insertó el tenedor }\end{array}$ \\
\hline \multicolumn{2}{|c|}{ Sujeción } & $\begin{array}{l}\text { Mediante un anillo } \\
\text { confeccionado con } \\
\text { el mismo termoplás- } \\
\text { tico en el cual se } \\
\text { introduce el pulgar } \\
\text { izquierdo }\end{array}$ & $\begin{array}{l}\text { Mediante abrojos } \\
\text { con el objeto de } \\
\text { que no se desplace } \\
\text { y pueda graduar el } \\
\text { ajuste a su pulgar } \\
\text { izquierdo. }\end{array}$ & $\begin{array}{l}\text { Mediante las escota- } \\
\text { duras, funcionalizar } \\
\text { la "tenaza" a través } \\
\text { de la F2 del pulgar } \\
\text { izquierdo y la por- } \\
\text { ción distal del mu- } \\
\text { ñón correspondiente } \\
\text { al quinto rayo }\end{array}$ & $\begin{array}{l}\text { Funcionalizando la } \\
\text { "tenaza" a través de la } \\
\text { F2 del pulgar izquierdo } \\
\text { y la porción distal del } \\
\text { muñón correspondien- } \\
\text { te al quinto rayo. }\end{array}$ \\
\hline \multirow[t]{2}{*}{ Mediciones } & $\begin{array}{l}\text { Respecto } \\
\text { al } \\
\text { usuario }\end{array}$ & $\begin{array}{l}\text { Longitud entre el } \\
\text { pulpejo del pulgar y } \\
\text { la porción distal del } \\
\text { muñón correspon- } \\
\text { diente al quinto rayo } \\
\text { (“C”, fig. 1). Circun- } \\
\text { ferencia del pulgar } \\
\text { izquierdo (“D”, fig. 1) }\end{array}$ & $\begin{array}{l}\text { Longitud entre el } \\
\text { pulpejo del pulgar y } \\
\text { la porción distal del } \\
\text { muñón correspon- } \\
\text { diente al quinto rayo } \\
\text { (“C”, fig. 2). Circun- } \\
\text { ferencia del pulgar } \\
\text { izquierdo (“D”, fig. 2) }\end{array}$ & $\begin{array}{c}\text { Longitud entre la } \\
\text { articulación IF del } \\
\text { pulgar izquierdo y el } \\
\text { apoyo carpiano ("D”, } \\
\text { fig. 3). La longitud de } \\
\text { la porción del mu- } \\
\text { ñón correspondiente } \\
\text { al quinto rayo (" } \mathrm{E}^{\prime} \text {, } \\
\text { fig. 3) }\end{array}$ & $\begin{array}{l}\text { Longitud entre la arti- } \\
\text { culación IF del pulgar } \\
\text { izquierdo y el apoyo } \\
\text { carpiano ("C", fig. 4). } \\
\text { La longitud de la por- } \\
\text { ción del muñón co- } \\
\text { rrespondiente al quinto } \\
\text { rayo (“D”, fig. 4) }\end{array}$ \\
\hline & $\begin{array}{c}\text { Respecto } \\
\text { al PA }\end{array}$ & $\begin{array}{l}\text { Largo del mango del } \\
\text { tenedor (“A", fig 1). } \\
\text { Circunferencia del } \\
\text { anillo (“B”, fig. 1). }\end{array}$ & $\begin{array}{l}\text { Largo del mango del } \\
\text { tenedor ("A", fig. 2). } \\
\text { Largo total de ambos } \\
\text { abrojos unidos ("B", } \\
\text { fig. 2) }\end{array}$ & $\begin{array}{l}\text { Diámetro del "flota } \\
\text { flota" ("A", fig. 3). } \\
\text { Longitud de esco- } \\
\text { taduras ("B", fig. 3). } \\
\text { Longitud total del } \\
\text { alto del "flota flota" } \\
\text { ("C", fig. 3) }\end{array}$ & $\begin{array}{c}\text { Longitud del tubo ("A", } \\
\text { fig. 4). Diámetro del } \\
\text { tubo ("B", fig. 4) }\end{array}$ \\
\hline \multicolumn{2}{|c|}{ Imagen ilustrativa } & Ver: Figura $N^{\circ} 1$ & Ver: Figura $N^{\circ} 2$ & Ver: Figura $N^{\circ} 3$ & Ver: Figura $N^{\circ} 4$ \\
\hline
\end{tabular}


Figura $\mathrm{N}^{\circ} 1$

-PA confeccionado en termoplástico liso Orfit

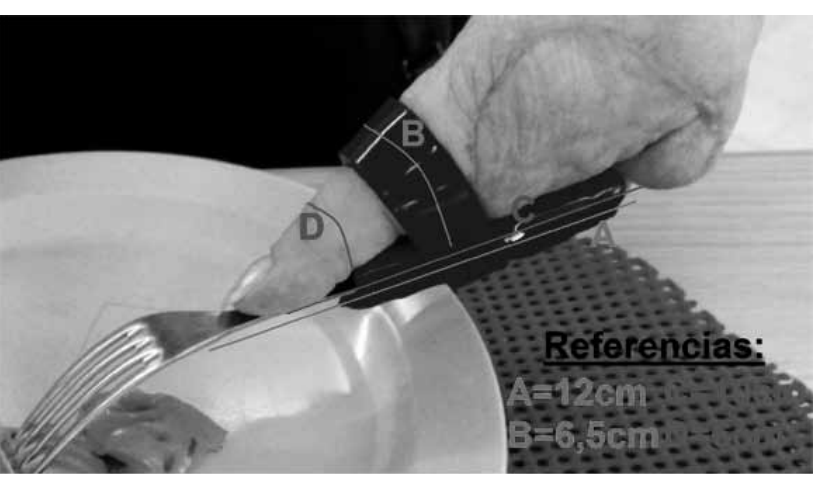

Figura $\mathrm{N}^{\circ} 2$

-PA confeccionado con espuma de polietileno

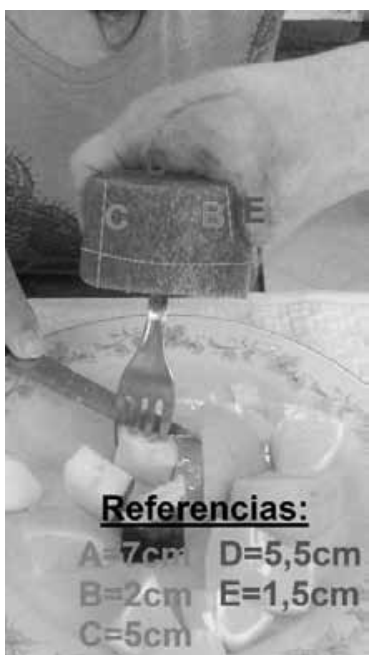

Figura $\mathrm{N}^{\circ} 3$

-PA confeccionado con material de descarte

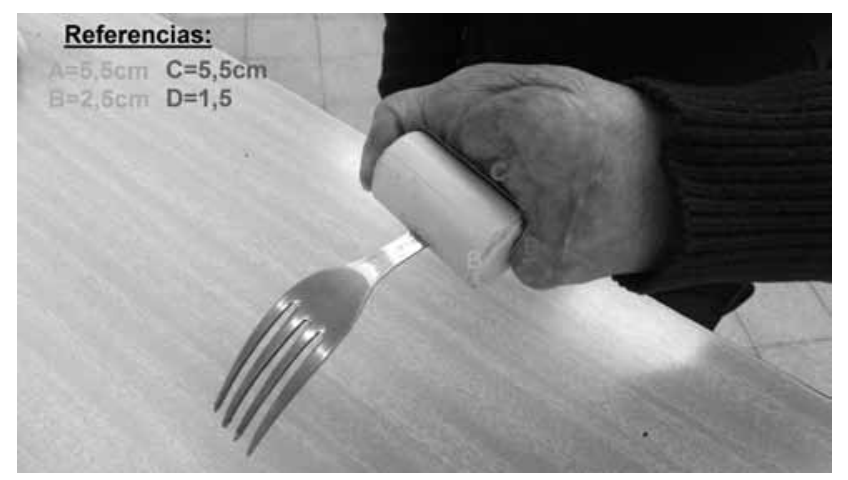

\section{Resultados}

Los PA incluyen cualquier producto, ya sea fabricado especialmente o disponible en el mercado, en este caso se valoraron los que fueron confeccionados, sus propiedades, ventajas y desventajas según los requerimientos del destinatario, pertinentes al área de las ABVD, más específicamente de alimentación.

Debido a la expresa demanda de GO de poder Ilevar a cabo en forma autónoma esta actividad, se fueron confeccionando una variada gama de opciones para lograr llegar a este fin.

El PA propuesto inicialmente, confeccionado en material termoplástico ORFIT liso azul, ofrecía la posibilidad de sostener el tenedor a través del anillo incorporado del diámetro de su pulgar izquierdo. El inconveniente que presentó este PA fue que en la prolongación de su uso se deslizaba, perdiendo el sostén que debía garantizar dado que el diámetro circunferencial del aniIlo, por la característica que brinda este material, quedaba en un diámetro fijado, no permitiendo graduarlo ni realizar ajustes en este sentido.

El diseño del segundo PA confeccionado con material ALL FIT de ORFIT, se selecciona a los efectos de conseguir que el anillo, en este caso de abrojo, esté fusionado al plástico (all fit) y garantice que no se produzcan movimientos ni se despegue del mango. De esta manera GO también lo iría ajustando y graduando a su necesidad.

En estos dos PA mencionados, GO utilizaba el tenedor, funcionalizando su pulgar izquierdo como apoyo a lo largo del mango, el flexor largo como refuerzo de "agarre" a nivel de la curva del tenedor y el segmento distal del muñón correspondiente al quinto rayo como tope. Por otra parte, con ambos PA no lograba llevar su porción de alimento a la boca dado que en el trayecto perdía el sostén del utensilio, por lo que debía utilizar su mano derecha para conseguir este fin.

En esta instancia GO manifiesta la inquietud de utilizar su muñón como "agarre" (a modo de "tenaza") prescindiendo de la sujeción y funcionalizando al máximo su estructura.

Por este motivo se plantea el tercer PA, confeccionado con material de espuma de polietileno (flota flota), al que GO tomaría con el pulgar izquierdo, más precisamente descargando la F2 en una de las escotaduras, y su porción distal del muñón correspondiente al quinto rayo en la otra escotadura realizada. De esta manera; con el tenedor incorporado en el centro del "flota-flota" asegurándonos que quede fijamente posicionado, se buscó 
garantizar que el utensilio no se desprenda del mango y que GO logre mantenerlo sostenido para orientarlo a la boca sin que se le caiga ante dicha intención.

Esta prueba se acercó mucho a la inquietud y satisfacción de GO, pero por etapas se le dificultaba el "agarre" que pretendía dado que el material ante su uso prolongado se le deslizaba y perdía el "agarre" mencionado.

Por lo detallado, se decide cambiar el diseño y material con la premisa de respetar la pretensión de GO.

Se resuelve utilizar un tubo de plástico para introducirle de manera perpendicular el tenedor al que se le cortó la longitud del mango. La toma la realizaría de ambos extremos del tubo, con su pulgar izquierdo y la longitud del muñón correspondiente al quinto rayo.

El Terapista Ocupacional desde su formación holística contribuye a mitigar el impacto ocasionado por un evento que pudiera estar contrariando el desempeño autónomo y cotidiano del individuo. Es así que en este caso clínico presentado se tomó con suma preponderancia la demanda de GO, logrando su satisfacción y confort en relación a los PA confeccionados, a la vez de alcanzar a optimizar la ejecución de sus funciones.

"No es lo que has perdido lo que cuenta, sino lo que haces con lo que queda". Mc Gonegal, C. (Hopkins \& Smith, 1998, p. 656).

\section{ReferenCias Bibliográficas}

Gutiérrez H., M. (2010). Módulo № 3: Antropometría y Biomecánica. Concepción, Chile: Unidad de Ergonomía, Facultad de Ciencias Biológicas, Universidad de Concepción.

Hopkins, H.L.; Smith, H.L. (1998): Terapia Ocupacional. Willard \& Spackman. 8va edición. Madrid, España: Ed. Panamericana.

Melo, J. L. (2009). Ergonomía Práctica: Guía para la evaluación ergonómica de un puesto de trabajo. Buenos Aires, Argentina: Fundación Mapfre.

Polonio L., B.; Durante M., P.; Noya A., B. (2001). Conceptos Fundamentales de Terapia Ocupacional. Ed. Panamericana. Madrid, España.

UNE-EN ISO 9999. (2007). Productos de apoyo para personas con discapacidad. Clasificación y terminología. (ISO 9999:2007). 4ta edición. BOE. España

\section{Documentos con acceso en el World Wide Web (WWW):}

Autores Varios. (2011). La Enciclopedia Libre Universal en Español. Calidad de Vida. Recuperado el 12 de julio de 2013, de http:// enciclopedia.us.es/index.php/Enciclopedia_Libre_Universal_ en_Espa\%C3\%B1ol 